\title{
ESTRATÉGIA DE CONTROLEBASEADA EM MODELO FENOMENOLÓGICO PARA UMA COLUNA DE DESTILAÇÃO CONTÍNUA EM BANCADA PARA A SEPARAÇÃO DE ETANOL-ÁGUA
}

\author{
A. APIO $^{1}$ e N. P. G. SALAU \\ Universidade Federal de Santa Maria, Departamento de Engenharia Química \\ E-mail para contato: ${ }^{1}$ andressaapio@ hotmail.com
}

\begin{abstract}
RESUMO - Um controlador PIDfoi proposto neste trabalho a fim de assegurar a concentração de etanol combustível próxima ao valor desejávelem um processo de destilação contínuo em bancada para separação da mistura etanol-água.O projeto de um sistema de controle, nesse quesito, pode assegurar a qualidade dos produtos com um menor consumo de energia. A modelagem fundamental do processo foi efetuada no software EMSO para esse fim, incluindo equações de conservação de massa e energia, além do modelo de equilíbrio de fasevan Laar. Os resultados permitiram a aquisição dos dados necessários mediante perturbações no processo para a posterior identificaçãodo sistema, realizada no softwareMatLab®. Para o controle da concentração de etanol no topo através da manipulação da potência na manta, o modelo paramétrico discreto Box-Jenkins foi o que melhor se ajustou e, por conseguinte, a melhor relação entre robustez e desempenho para o projeto decontrolador foi obtida pelo método de sintonia baseado na minimização da integral do erro absoluto ponderado no tempo (do inglês,Integral Time AbsoluteError- ITAE).
\end{abstract}

\section{INTRODUÇÃO}

O etanol, segundo Baiet al. (2008), é considerado um dos mais importantes combustíveis renováveis devido aos benefícios econômicos e ambientais do seu uso, sendo uma boa alternativa para substituir o petróleo. O processo de produção do etanol combustível compreende desde a fermentação da matéria prima até a operação unitária de destilação. De acordo com a ANP (2012), o teor alcoólico para o álcool combustível hidratado deve ficar acima de 92,5 $5^{\circ}$ INPM $(83,04 \mathrm{~mol} \%)$.

As colunas de destilação para a separação binária etanol-água, no entanto, demandam um grande gasto energético, trazendo a necessidade de projetar sistemas de controle que garantam a qualidade dos produtos com um consumo mínimo de energia. A estrutura de controle pode ser obtida pelo modelo do processo, que é baseado nas propriedades físicas do sistema, através de equações de conservação de massa, energia e quantidade de movimento. 


\section{9 a 22 de outubro de 2014 \\ Florianópolis/SC}

O controle de colunas de destilação tem a função de manter a operação da coluna de acordo com a estratégia de produção adotada pelo operador, ou seja, no valor de concentração de etanol desejado. Assim, através da relação entre as variáveis controladas e manipuladas, propõe-se a utilização do algoritmo de controle e do método de sintonia mais adequados, satisfazendo requisitos de desempenho, robustez e estabilidade frente a eventuais distúrbios que possam ocorrer na planta.

\section{MODELAGEM DO PROCESSO DE DESTILAÇÃO}

A modelagem do processo de destilação contínuo foi baseadano modelo apresentado porLuyben (1996), considerando a transferência de energia no topo e no fundo da coluna. Esse modelo foi adaptado para uma coluna de destilação em bancada, localizada na Universidade Federal de Santa Maria, cujo trabalho foi proposto por Mayer (2010).Portanto, a determinação da composição da fase líquida em cada estágio é calculada através do balanço de massa e a temperatura é calculada pelo balanço de energia (refervedor e pré-condensador) com o auxílio do modelo de coeficiente de atividade de van Laar.Para o seguinte modelo, algumas considerações são feitas, como acúmulo da fase vapor em cada estágio desprezível, acúmulo molar da fase líquida constante nos pratos intermediários e no condensador, condensador total, perdas de energia desprezíveis, vazões internas de líquido e vapor constantes ao longo da coluna, pressão de operação constante, queda de pressão ao longo da coluna desprezível e mistura perfeita da fase líquida e da fase vapor.

As equações do modelo representam o cálculo diferencial das composições no destilador, nos estágios intermediários e no condensador. As composições molares das frações líquida e vapor são $x(j, i)$ e $y(j, i)$, respectivamente, onde ' $j$ ' refere-se ao componente, e ' $i$ ' refere-se ao estágio de equilíbrio. Neste caso, o componente 1 é o etanol, e o 2 a água. Já os estágios de equilíbrio compreendem desde o destilador ( $12^{\circ}$ estágio) até o condensador ( $1^{\circ}$ estágio). $V$ é a vazão de vapor da mistura etanol-água que é a formada a partir do destilador, em $\mathrm{mol} / \mathrm{min}$, F é a vazão de alimentação na fase líquida, em mol/min. $M_{B}$ é o acúmulo molar no $12^{\circ}$ estágio (destilador), assim como $M_{S}$ é o acúmulo molar nos estágios intermediários e $M_{C}$ é o acúmulo molar no condensador. Ainda, $R$ é o valor da razão de refluxo, $F S$ é o estágio de alimentação e $N S$ é o número de estágios. Tendo em vista que neste estudo não são consideradas variações na pressão e no volume, ou seja, o trabalho realizado é nulo, os balanços de energia são calculados somente em termos de entalpias da mistura etanol-água tanto para a fase líquida, designada por $h^{l}(i)$, quanto para a fase vapor, designada por $h^{v}(i)$. As demais equações do modelo se resumem em equações diferenciais de balanço de massa e de energia, bem como a equação global da coluna, conforme a seguir:

O balanço para o condensador $(i=1)$ é dado pela equação 1:

$$
\frac{d x(j, i)}{d t}=\frac{V}{M_{C}} \cdot(y(j, i+1)-x(j, i))
$$


O balanço para o pré-condensador $(i=2)$ é dado pela equação 2 e o balanço de energia pela equação 3:

$$
\begin{aligned}
& \frac{d x(j, i)}{d t}=\frac{V}{M_{S}} \cdot(y(j, i+1)-y(j, i))+\frac{V}{M_{S}} \cdot \frac{R}{(R+1)} \cdot(x(j, i-1)-x(j, i)) \\
& \frac{d H_{\text {cond }}}{d t}=\frac{V}{M_{S}} \cdot\left(h^{v}(j, i+1)-h^{v}(j, i)\right)+\frac{V}{M_{S}} \cdot \frac{R}{(R+1)} \cdot\left(h^{l}(j, i-1)-h^{l}(j, i)\right)+Q_{\text {cond }}
\end{aligned}
$$

O balanço para a seção de retificação(de $i=3 \mathrm{a} F S$ - 1$)$ é dado pela equação 4:

$$
\frac{d x(j, i)}{d t}=\frac{V}{M_{S}} \cdot(y(j, i+1)-y(j, i))+\frac{V}{M_{S}} \cdot \frac{R}{(R+1)} \cdot(x(j, i-1)-x(j, i))
$$

O balanço para a seção de alimentação $(i=F S)$ é dado pela equação 5:

$$
\begin{aligned}
\frac{d x(F S)}{d t}=\frac{V}{M_{S}} \cdot(y(j, F S+1)-y(j, F S))+\frac{V}{M_{S}} \cdot \frac{R}{(R+1)} \cdot(x(F S-1)-x(F S))+\frac{F}{M_{S}} & \cdot(x(F S)-z)
\end{aligned}
$$

O balanço para a seção de esgotamento $($ de $i=F S+1$ a $N S-1)$ é dado pela equação 6 :

$$
\begin{gathered}
\frac{d x(j, i)}{d t}=\frac{V}{M_{S}} \cdot(y(j, i+1)-y(j, i))+\frac{V}{M_{S}} \cdot \frac{R}{(R+1)} \cdot(x(j, i-1)-x(j, i))+\frac{F}{M_{S}} \\
\cdot(x(j, i-1)-x(j, i))
\end{gathered}
$$

O balanço para o destilador (i=NS) é dado pela equação 7 e o balanço de energia pela equação 8:

$$
\begin{aligned}
& \frac{d x(j, i)}{d t}=\frac{V}{M_{B}} \cdot(x(j, i)-y(j, i))+\frac{V}{M_{B}} \cdot \frac{R}{(R+1)} \cdot(x(j, i-1)-x(j, i))+\frac{F}{M_{B}} \cdot(x(j, i-1)-x(j, i)) \\
& \frac{d H_{d e s t}}{d t}=\frac{V}{M_{B}} \cdot\left(h^{l}(j, i)-h^{v}(j, i)\right)+\frac{V}{M_{B}} \cdot \frac{R}{(R+1)} \cdot\left(h^{l}(j, i-1)-h^{l}(j, i)\right)+\frac{F}{M_{B}} \\
& \cdot\left(h^{l}(j, i-1)-h^{l}(j, i)\right)+Q_{\text {dest }}
\end{aligned}
$$

As quantidades de energia trocadas no destilador $\left(Q_{\text {dest }}\right)$ e no pré-condensador $\left(Q_{\text {cond }}\right)$ são calculadas pelas equações 9 e 10, onde $V_{\text {water }}$ e $V_{\text {vapor }}$ são as vazões de água no trocador de calor do pré-condensador e de vapor na serpentina de aquecimento do destilador, respectivamente. Ainda, as variações de energia são calculadas em termos de entalpias, sendo $\Delta H_{\text {vapora variação da }}$ entalpia no destilador e $\Delta H_{\text {water }}$ a variação de entalpia da água de resfriamento no précondensador.

$$
Q_{\text {cond }}=V_{\text {water }} \cdot \Delta H_{\text {water }}
$$




$$
Q_{\text {dest }}=V_{\text {vapor }} \cdot \Delta H_{\text {vapor }}
$$

Por fim, o cálculo de equilíbrio entre as fases é feito mediante relação de equilíbrio representada na equação 11, onde a fugacidade na fase líquida é estimada a partir do coeficiente de atividade $(\gamma(j, i))$, calculadapelas equações12 e 13 do modelo de van Laar, pela pressão de vapor saturado $\left(P_{S A T}(j, i)\right)$ e pela pressão do sistema $(P)$. Considera-se a fase vapor como ideal $\left(\phi^{\text {Vapor }}(j, i)=1\right)$.

$$
\begin{aligned}
& y(j, i)=x(j . i) \cdot \frac{P_{S A T}(j, i)}{P} \\
& \ln \gamma_{1}=A_{12}\left(\frac{A_{21} x_{2}}{A_{12} x_{1}+A_{21} x_{2}}\right)^{2} \\
& \ln \gamma_{2}=A_{21}\left(\frac{A_{12} x_{1}}{A_{12} x_{1}+A_{21} x_{2}}\right)^{2}
\end{aligned}
$$

Onde os parâmetros $\mathrm{A}_{12}$ e $\mathrm{A}_{21}$ do modelo de van Laar para a mistura binária etanol-água são dados por Perry (1999):

$$
A_{12}=1.6798 \text { e } A_{21}=0.9227
$$

\section{MODELO IDENTIFICADO DO PROCESSO}

O primeiro passo para análise do processo se consistiu em avaliar a resposta do modelo fenomenológico proposto a perturbações positivas e negativas nas variáveis manipuladas, no caso, perturbação do tipo sinal randômico pseudo-binário (do inglês, pseudorandombinarysignalPRBS). Como se trata da destilação contínua da mistura etanol-água, a variável controlada é a composição de topo, e a variável manipulada é a potência da manta de aquecimento.

A simulação do modelo fenomenológico foi realizada através do software EMSO para obter a resposta da composição de topo frente à perturbação realizada na potência da manta de aquecimento, conforme observado na Figura 1. Posteriormente foi utilizada a caixa de ferramentas de identificação de sistemas (System Identification Toolbox) do software MatLab®, que permitiu a criação do modelo matemático do sistema dinâmico por meio dos dados gerados pelo modelo fenomenológico. Quatro estruturas básicas de modelos discretos foram utilizadas para a identificação de modelos dinâmicos, como os modelos Output-Error (OE), AutoRegressivemodelwithaneXternal input(ARX), AutoRegressiveMovingAverageModelwitheXogenous inputs (ARMAX) e Box-Jenkins(BJ) com polinômios de segunda ordem. Esses modelos discretos foram convertidos em modelos contínuos, obtendo-se a função de transferência do processo para cada um dos modelos, conforme apresentado na Tabela 1 . O resultado mais satisfatório foi encontrado com o modelo BJ, com um ajuste de $93,66 \%$. 

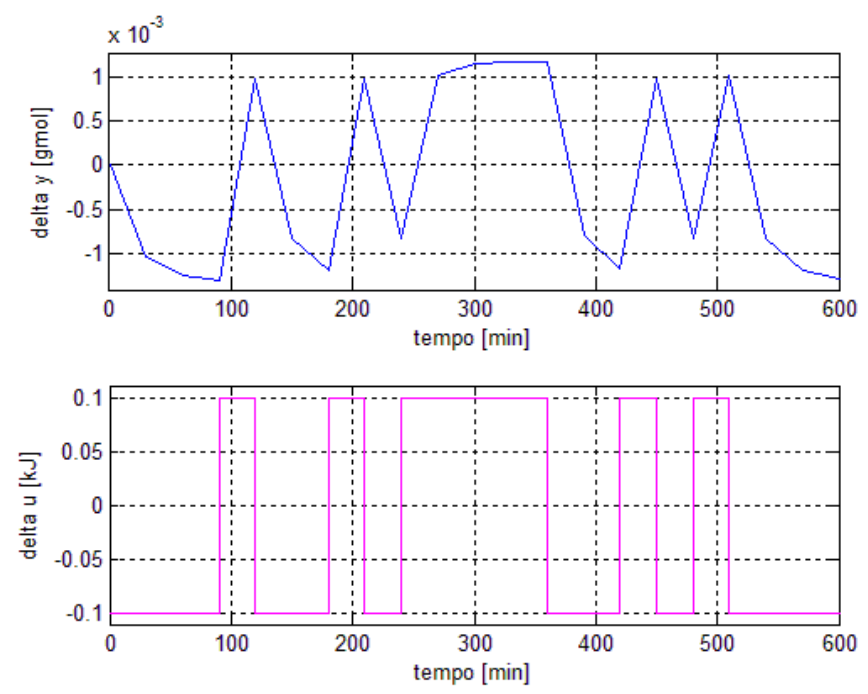

Figura 1 - Perturbação PRBS realizada no simulador EMSO: delta y refere-se à variação da composição do topo e delta u refere-se à variação da potência da manta de aquecimento.

Tabela 1 - Identificação dos modelos dinâmicos

\begin{tabular}{|c|c|c|}
\hline Função de transferência & Modelo discreto & Ajuste (\%) \\
\hline$G(s)=\frac{0,02134 s+0,0286}{s^{2}+2,73 s+2.179}$ & BJ22221 & 93,66 \\
\hline$G(s)=\frac{0,02214 s+0,02029}{s^{2}+2,73 s+2,179}$ & OE221 & 93,29 \\
\hline$G(s)=\frac{0,02214 s+0,02029}{s^{2}+2,544 s+1,671}$ & ARX221 & 93,03 \\
\hline$G(s)=\frac{0,01768 s+0,7329}{s^{2}+4,514 s+5,721}$ & AMX2221 & 92,04 \\
\hline
\end{tabular}

\section{MODELO DE SINTONIA DOS CONTROLADORES}

Neste trabalho foi escolhido o controlador do tipo proporcional integral derivativo (PID), por ser o mais utilizado em diferentes tipos de controle de processos e principalmente devido a sua simplicidade. Foram comparados quatro métodos de sintonia para controladores PID: InternalModelControl (IMC), ITAE, Kappa-Tau e Ziegler-Nichols. Através desses métodos foram realizadas simulações da sintonia do controlador, além da avaliação do comportamento do controlador PID sintonizado por cada método frente às perturbações degrau unitário no set-point 
e distúrbio na carga. Por conseguinte, a simulação dos métodos de sintonia gerou os parâmetros do controlador para cada método, conforme apresentado na Tabela 2.

Tabela 2 - Resultados dos parâmetros de sintonia, tempo de assentamento e sobre-elevação para cada método de sintonia

\begin{tabular}{|c|c|c|c|c|c|}
\hline $\begin{array}{c}\text { Método de } \\
\text { Sintonia }\end{array}$ & Kp & Ti & Td & $\begin{array}{c}\text { Tempo de } \\
\text { assentamento } \\
(\mathbf{m i n})\end{array}$ & $\begin{array}{c}\text { Sobre-elevação } \\
(\%)\end{array}$ \\
\hline IMC & 39,968 & 0,9770 & 0,2428 & 5,5085 & 0,0011 \\
\hline ITAE & 51,066 & 1,0008 & 0,3992 & 2,4401 & 0,0010 \\
\hline Kappa-Tau & 13,666 & 0,5262 & 0,1094 & 7,4264 & 0,0011 \\
\hline Ziegler-Nichols & 43,589 & 2,0843 & 0,5211 & 20,5020 & 0,0392 \\
\hline
\end{tabular}

Nas Figuras 3 e 4 observa-se que os métodos de sintonia apresentaram comportamento estável após as perturbações, afirmando a capacidade do controlador proposto de manter controlada a concentração do topo do processo de destilação. Além disso, o comportamento mais satisfatório para o projeto de controlador PID foi obtido com a sintonia do método ITAE, devido ao seu baixo tempo de assentamento e pequena sobre-elevação.
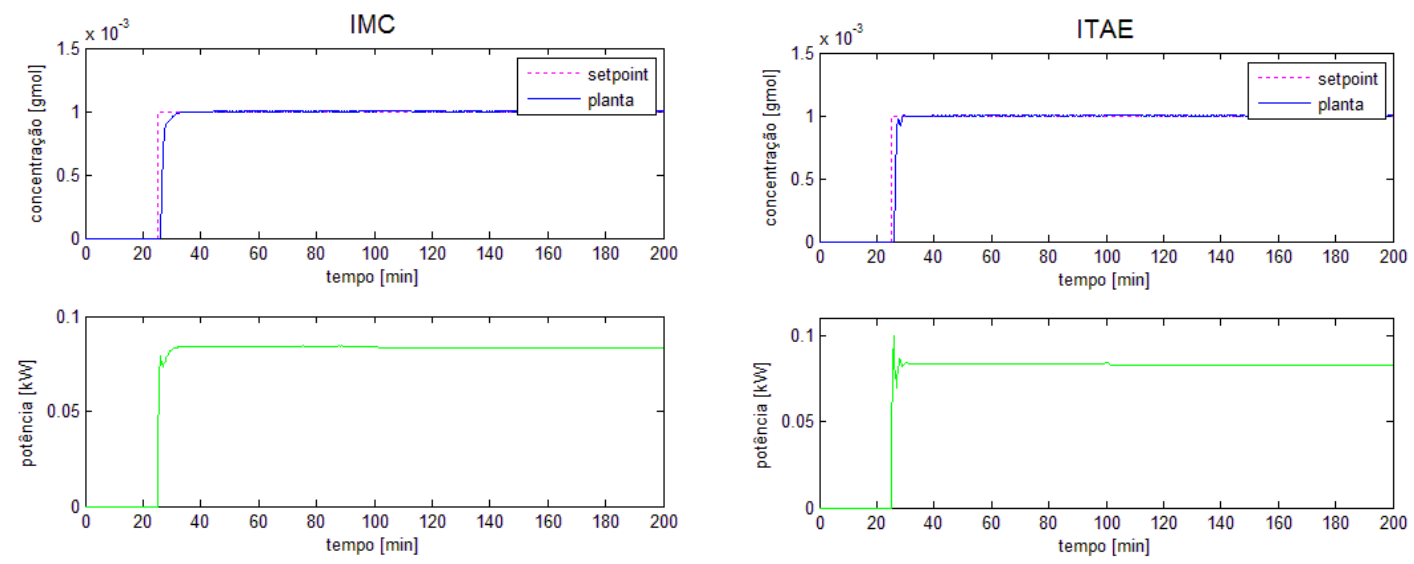

Figura 3 - Resposta do controlador para uma mudança no set-point da concentração de topo e frente a um distúrbio na potência fixa para os métodos de sintonia IMC e ITAE. 

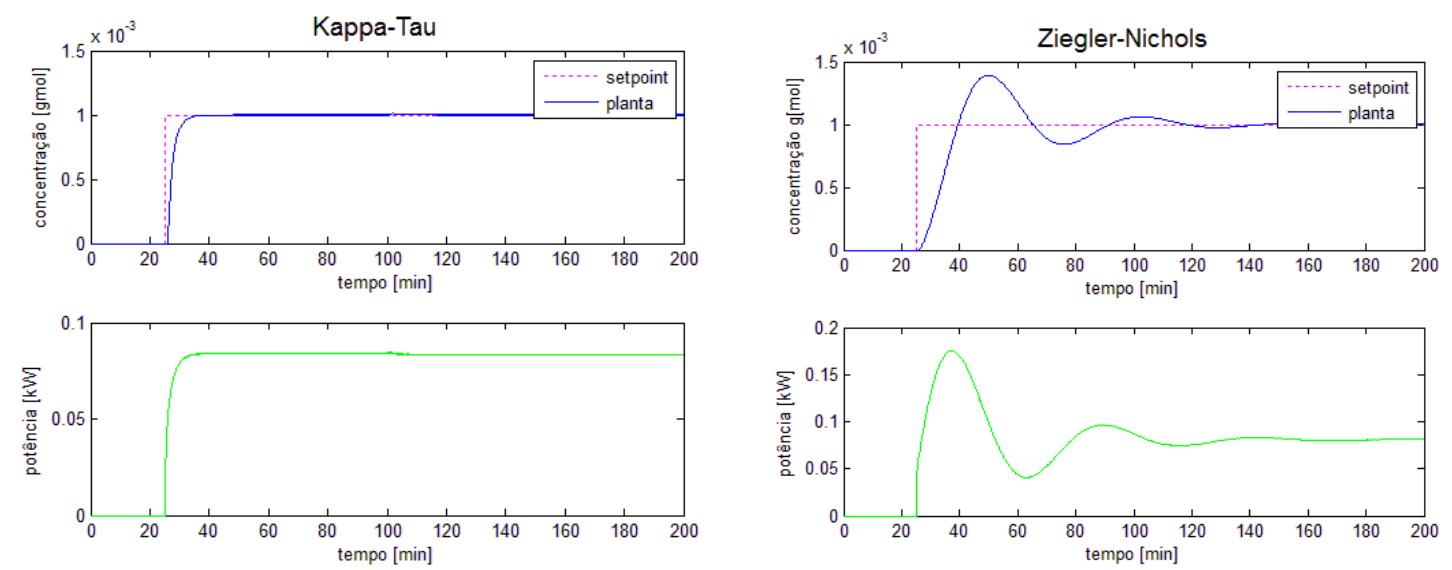

Figura 4 - Resposta do controlador para uma mudança no set-point da concentração de topo e frente a um distúrbio na potência fixa para os métodos de sintonia Kappa-Tau e Ziegler-Nichols.

\section{ANÁLISE DO DESEMPENHO DO CONTROLADOR}

Para afirmar qual a melhor sintonia do controle de PID em questão, a análise gráfica não é o suficiente. Por isso, foram calculados os índices de desempenho e robustez para cada método. Os índices de desempenho foram determinados através dos métodos da integral do erro absoluto (IAE), integral do erro quadrático (ISE) e integral do erro absoluto ponderado no tempo (ITAE). Já os índices de robustez são determinados pela margem de ganho (MG), reserva de fase (RF) e máxima sensibilidade (Ms). Os resultados podem ser visualizados na Tabela 3.

Tabela 3 - Índices de desempenho e robustez do controlador PID proposto para cada método de sintonia

\begin{tabular}{|c|c|c|c|c|c|c|}
\hline \multirow{2}{*}{$\begin{array}{c}\text { Método de } \\
\text { Sintonia }\end{array}$} & \multicolumn{2}{|c|}{ Índices de Desempenho } & \multicolumn{3}{|c|}{ Índices de Robustez } \\
\cline { 2 - 7 } & IAE & ISE & ITAE & $\mathbf{M}_{\mathbf{S}}$ & MG & RF \\
\hline IMC & 0,0021 & 0 & 0,0546 & 1,5077 & 3,028 & 75,2582 \\
\hline ITAE & 0,0017 & 0 & 0,0451 & 1,8866 & 2,1132 & 74,2882 \\
\hline Kappa-Tau & 0,0029 & 0 & 0,0810 & 1,3118 & 5,2848 & 73,5742 \\
\hline Ziegler-Nichols & 0,0188 & 0 & 0,9653 & 1,8510 & 1,9240 & 99,2181 \\
\hline
\end{tabular}

O controlador sintonizado pelo método ITAE apresentou todos os valores para o índice de robustez dentro dos limites desejados, além de apresentar valores satisfatórios para o índice de desempenho. Além disso, esse método de sintonia garante uma boa associação entre desempenho e robustez. 


\section{CONCLUSÃO}

A fim de estudar a etapa de purificação do etanol até as especificações do etanol combustível hidratado, construiu-se um modelo de coluna de destilação, baseado em uma coluna de destilação contínua localizada na Universidade Federal de Santa Maria. Esse modelo envolveu um conjunto de equações diferenciais referentes aos balanços de massa e de energia em cada estágio de equilíbrio, além das demais equações algébricas do sistema. Quanto ao modelo termodinâmico utilizado para o equilíbrio líquido-vapor em cada estágio, foi escolhida a equação de van Laar para o cálculo do coeficiente de atividade do líquido juntamente ao modelo ideal para a mistura vapor.

Posteriormente, elaborou-se uma estratégia de controle para esse modelo fenomenológico, onde foi escolhida a variável controlada como a composição de topo, e a variável manipulada como a potência da manta de aquecimento. Tendo como objetivo representar a resposta da concentração mediante a perturbação na variável manipulada, utilizou-se o processo de identificação de modelos discretos. O modelo BJ (Box-Jenkins) foi, então, o que melhor se ajustou aos dados obtidos através da simulação do modelo. Para a sintonia do controlador PID projetado, o melhor resultado obtido através dos critérios de desempenho avaliados foi para o método de sintonia ITAE, cuja proposta é a de melhor compromisso entre o desempenho e a robustez do controlador.

\section{REFERÊNCIAS}

ANP - Agência Nacional do Petróleo, Gás Natural e Biocombustíveis. (2005). Resolução ANP $\mathrm{n}^{\circ}$ 56, de 06 de dezembro de 2005. Disponível em <http://www.anp.gov.br>. AccessadoemJunho de 2012.

BAI, F.W.; ANDERSON, W.A.; MOO-YOUNG, M. Ethanol fermentation technologies from sugar and starch feedstocks. Biotechnology Advances, 26, p. 89-105, 2008.

LUYBEN, W. L. Process Modeling, Simulation and Control for Chemical Engineers.2nd Edition.Chemical Engineering Series.McGraw-Hill International Editions, New York, 1996.

MAYER, F. D. Desenvolvimento da tecnologia de destilação apropriada à produção de álcool combustível em pequena escala. Dissertação de Mestrado PPGEPRO, Universidade Federal de Santa Maria, Santa Maria, 2010.

PERRY, R. H. Chemical Engineering Handbook. 7th edition. McGraw-Hill, New York, 1999. 